\title{
Design and implementation of advanced systems in a flexible-stretchable technology for biomedical applications
}

\author{
R. Carta ${ }^{a}, *$ P. Jourand ${ }^{a}$, B. Hermans ${ }^{a}$, J. Thoné ${ }^{a}$, D. Brosteaux ${ }^{b}$, T. Vervust ${ }^{b}$, F. Bossuyt $^{b}$, \\ F. Axisa ${ }^{\mathrm{b}}$, J. Vanfleteren ${ }^{\mathrm{b}}$, R. Puers ${ }^{\mathrm{a}}$ \\ a K.U. Leuven, ESAT-MICAS, Kasteelpark Arenberg, B-3001 Leuven, Belgium \\ b IMEC/CMST, Ghent, Belgium
}

\section{A R T I C L E I N F O}

\section{Article history:}

Received 27 September 2008

Received in revised form 3 March 2009

Accepted 3 March 2009

Available online $\mathrm{xxx}$

\section{Keywords:}

Flexible-stretchable electronics

Wireless power supply

Textile-embedded systems

\begin{abstract}
A B S T R A C T
This paper presents the design and implementation of an advanced system on flexible and stretchable technology. The technology platform consists of a matrix of flexible non-stretchable functional islands linked together by a net of elastic interconnections. Several technologies have been developed and tested in the design of simplified demonstrators before studying the design of an advanced system. The target system is a wireless battery charger which supplies power and supports bidirectional data transfer during recharge. The system is intended to serve as a general purpose platform for biomedical parameter monitoring and the design is focused on the embedding in clothes.
\end{abstract}

(C) 2009 Elsevier B.V. All rights reserved.

\section{Introduction}

Portable electronic systems have become typical components in everyday life. The target applications in this paper are in the field of biomedical parameter monitoring [1-5] and smart clothing that improve the safety of workers in harsh environments [1,2] form the focus of the created system. The presence of these systems should be hardly noticeable to the user. One way to improve comfort and portability is to embed them in textile; another is the use of fully flexible integrated carriers. The combination of the two strategies implies the need for elastic substrates which provide a waterproof encapsulation and guarantee good adhesion to the textile fibers. The adoption of a flexible substrate does not necessarily guarantee the flexibility of the entire electronic circuit. The layout and the placement of the constituent components of the circuit, on such a substrate, are crucial to preserve the envisaged applications. Since components are intrinsically rigid, a too dense or not wellorganized layout may drastically limit the flexibility of the overall structure. Design efforts should focus in a routing where rigid components form an ordinate grid and their proximity is limited in order not to affect the overall flexibility. However, system flexibility does not imply stretchability. In fact, even if the substrate is stretchable, the routing lines are intrinsically not, unless a dedicated design is adopted. Our proposed approach, aiming the realization of flexible-

\footnotetext{
* Corresponding author. Tel.: +32 16321108; fax: +32 16321975.

E-mail address: riccardo.carta@esat.kuleuven.be (R. Carta).
}

stretchable electronic systems, consists of a network of flexible non-stretchable islands connected by stretchable joints [6-9]. In this way an overall flexible-stretchable electronic circuit can be achieved by composing a set of non-stretchable functional units. Common SMD components, platinum plated electrodes, antennas and flexible batteries can be incorporated [6]. Some systems have been presented that use wireless power supplies but often require external electronics in close proximity for control and power supply $[1,2]$. The system presented in this paper takes advantage of a combination of an inductive link and a rechargeable battery. The inductive link is meant to recharge the battery when the system is not in use (e.g. overnight). During battery recharge, bidirectional data transfer is supported in order to tune the system parameters and download stored data (e.g. biological parameters) from the textile-embedded acquisition system. Several simplified demonstrators have been developed to test the technological feasibility. An advanced system has been designed, and is currently in production on flexible-stretchable technology $[6,10]$.

\section{Design and implementation}

A good understanding of the technology limitations is fundamental in order to succeed in the design. A standard PCB design can be transferred to a silicone substrate only when some essential adaptations are carried out.

Firstly, the ground plane is missing and the dielectric properties of silicone may change depending on temperature variations and moisture exposure [11,12]: silicone elastomers are in fact 


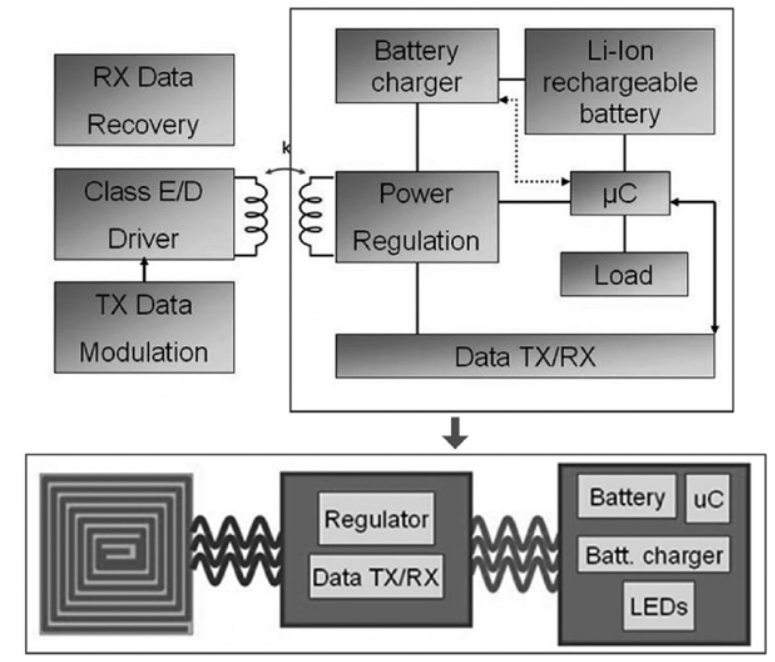

Fig. 1. Block level schematic of the wireless battery charging system (top) and its implementation on flexible-stretchable substrate (bottom), consisting of three flexible substrates, interconnected with stretchable paths.

easily penetrated by water and contaminated by ions [12]. This is especially an issue in case of remote powered systems relying on inductive links. To compensate the effect of varying substrate losses, thicker metal routing is used in the design of coil antennas.

Secondly, current stretchable circuit technologies at CMST allow for one metal layer only; for conductor crossovers one needs to use $0 \Omega$ cross-over resistors in the stretchable circuit part. An alternative is to realize a multilayer structure on a standard rigid or flexible circuit interposer, which is then mounted as a component on the stretchable circuit. With this approach, even a single layer design can achieve complex interconnects. The drawbacks are an extended assembly time and a larger layout compared to a two layer design.

The concept underlying the design is illustrated in Fig. 1. On the top, a block level schematic of the developed system is depicted. The left and right parts are respectively the external base and the embedded battery charger which are connected by the inductive link. A microcontroller (MCU) monitors the battery status, manages the communication process and drives the load. For demonstration purposes, the load consists of a LED array. In a future stage, their place will be taken by sensors for biomedical parameter monitoring and a memory module for data storage. Research is ongoing to integrate heart beat and respiration rate monitoring systems. The heart beat can be extracted from ECG measurements [13] or by applying pulse oximetry techniques [14]. The respiration rate can be monitored by detecting the resistive, capacitive or inductive variation of a conductive ribbon (e.g. conductive silicone) wound around the patient's chest. These changes are related to the ribbon extension/contraction cycle occurring during the respiration activity. A combination of a few parameters can be used to get better read-out when the variation of a single parameter is not large enough to allow a clear detection. A similar approach was developed and tested in textile [13] and the implementation on silicone based technologies is now under investigation.

The circuitry is meant to be implemented on a hermetically sealed network of flexible islands connected by elastic interconnections $[6,10]$. The number of islands is application-dependent and can theoretically be expanded to a large web. In the presented work, a configuration based on three islands is used. The first island only contains a planar coil that can eventually be replaced by an embroided one [13]. Power regulation and data communication sub-modules are located on the second island. DC power is distributed to electronics on other islands. A third island contains an ultra thin rechargeable battery, a MCU, a battery charger and the load.

\subsection{System design}

Fig. 2 depicts a simplified schematic of the developed system. The circuit can be divided in three functional blocks: the power unit, the telemetry unit and the control block. The power module includes an inductive link with its magnetic field source at the primary side, a power receiving/converting circuit at the secondary side, a battery charger and a rechargeable lithium cell. The inductive link provides power for the battery charge and electronics during the charging operation. The link is driven by a dedicated class $\mathrm{E}$ driver and the induced alternating field is partially picked up by a parallel resonant LC tank at the secondary side. A doubling configuration rectifier was chosen to improve the link robustness to misalignments and decoupling. A two-stage voltage regulation provides $5 \mathrm{~V}$ to the battery charger and $3.3 \mathrm{~V}$ for the controller and the rest of the electronics. Two low drop-out (LDO) regulators in a SOT23 package are used. They only require stabilizing capacitors as external components. The battery charging is handled by a dedicated IC (LTC4054l) by Linear Technology which also comes

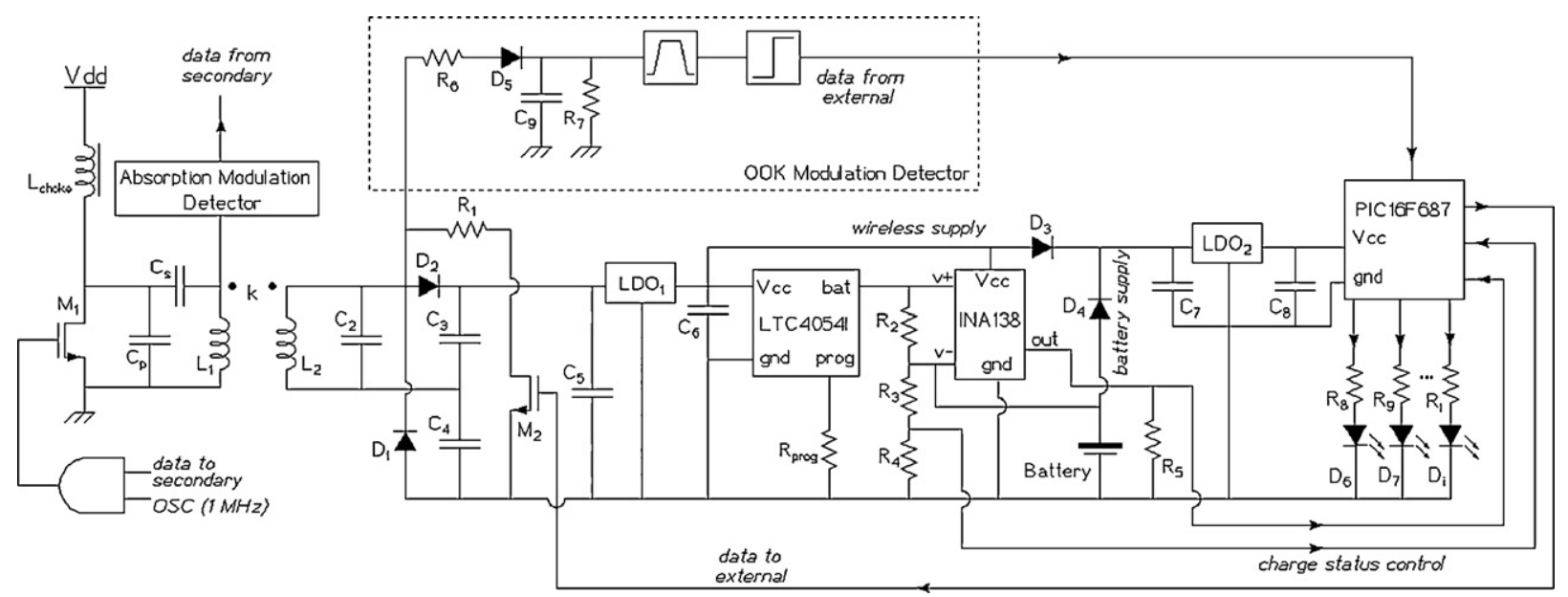

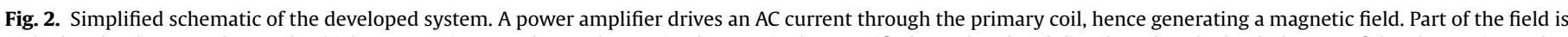

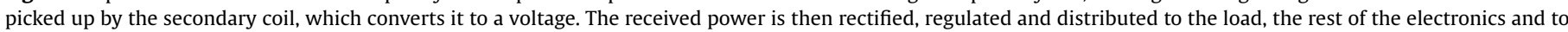
the battery charger. The system also supports bidirectional data communication using OOK modulation in the downlink and absorption modulation in the uplink. 


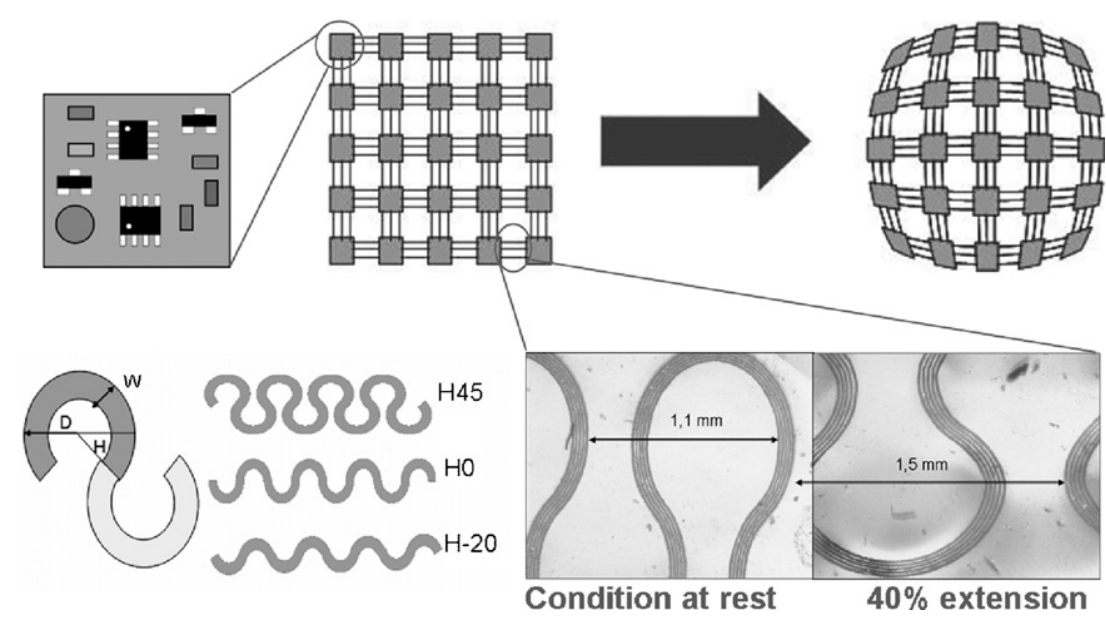

Fig. 3. Principle of stretchable electronics (top) and characterization of horse shoe shaped stretchable interconnections (bottom) [18].

in a SOT23 package and only requires a few external components. Its large dynamic range in charging current, from 10 to $150 \mathrm{~mA}$, makes this product suitable for wireless battery charge applications. The charging current can be set easily by changing the resistor

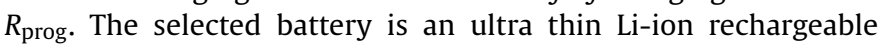
cell (PGEB0053559) which has $3.7 \mathrm{~V}$ nominal voltage and $65 \mathrm{mAh}$ capacity. The battery thickness, a mere $500 \mu \mathrm{m}$, makes this component the perfect candidate for this application. A charging current of $30 \mathrm{~mA}$ was set in order to achieve a full charge in a few hours, lower rates or larger cells can be implemented as well. The backbone of the above described control block is a MCU (PIC16F687). The monitoring of the charge is performed in combination with a shunt monitor (INA138) which provides the battery's charging current to the MCU. Information about the battery voltage is directly available to the MCU through a voltage divider. These signals are then digitized and stored in temporary registers awaiting transmission. A second LDO is required to provide a constant voltage to the MCU in both wireless and battery supplies. The charger and the shunt require $5 \mathrm{~V}$ to work properly. Since this level cannot be achieved with a $3.7 \mathrm{~V}$ battery and these components do not need any bias in battery mode, the MCU supply was set to $3.3 \mathrm{~V}$. In this way full operation is guaranteed also when the battery starts its discharging cycle. Diodes $\mathrm{D}_{3}$ and $\mathrm{D}_{4}$ are required to prevent the charger and the shunt to be biased while the system is battery powered, and also, to avoid any current draw from the battery during recharge. The telemetry module supports bidirectional data transfers at $4.8 \mathrm{kbps}$. Power and data share the same channel, through the inductive link. Although several kinds of modulation are possible, On-Off-Keying (OOK) modulation for the downlink (primary to secondary side) and absorption modulation [1] for the uplink are selected. The OOK modulation is performed by directly acting on the gate of $\mathrm{M}_{1}$; data are recovered at the secondary side with the cascade of an envelope detector, a filter stage and a comparator before being processed by the MCU. Absorption modulation is achieved by changing the equivalent impedance at the primary side, through actuation of the switch $\mathrm{M}_{2}$. This variation can be detected at the primary side in several ways: detecting the voltage drop across the coil $\mathrm{L}_{1}$ with a high resistance (or capacitive divider) or detecting the current drop with a small transformer in series with $\mathrm{L}_{1}[1,15]$. In both cases the data detection should not affect the driver behavior. Data demodulation is performed again with an envelope detector and a comparator. In order to maximize the bandwidth in both directions, a master-slave communication strategy was defined with the master located at the primary side.

The telemetry module is controlled by a PC through a serial port in the external base.

\section{Technologies for flexible-stretchable interconnections}

In a flexible-stretchable electronic circuit, the interconnection network should be elastic. For the realization of elastic interconnections, innovative work was recently performed by researchers from Princeton University [8,16], where elastic joints are achieved by electron-beam evaporation of a $100 \mathrm{~nm}$ thick gold film on an elastic polydimethylsiloxane (PDMS) substrate. Drawbacks of this technique are the restriction to extremely thin gold films, the need for high cost equipment and for non-standard assembly technologies on those substrates. The resulting skin-like electronic circuits are useful for electronic skin applications. Another implementation contains a microelectrode array for monitoring the neuron-electrical activity in the brain [16]. Our work in the field of elastic electronics is based on the realization of electrical interconnections by $2 \mathrm{D}$ spring shaped metallic tracks, which are embedded in an elastic polymer membrane. Conventional PCB manufacturing process flows are used to achieve these interconnections.

In Section 3.1, the basic shapes for flexible and stretchable technologies are discussed. Sections 3.2 and 3.3 describe two possible technologies to obtain these patterns completely embedded in silicone. The process flow of the production of the demonstrators, explained in Sections 4.1 and 4.2, refers to these two technologies.

\subsection{Geometry of the interconnections}

Fig. 3 depicts the idea underlying the realization of stretchable interconnections. The function of the elastic joints is of paramount importance in order to give stretchability to a structure composed by non-stretchable elements.

The goal is to create elastic interconnections which can support at least $50 \%$ and up to $100 \%$ elongation. Although the required elongation in the target application will probably stay below $30 \%$, the $100 \%$ target guarantees a better reliability. Moreover, aiming at washable electronics embedded in textile implies that the systems should be able to survive harsh environments (crumpling, twisting, etc.), so that elongations close to $100 \%$ might be needed.

Finite element analysis (FEA) has been used to characterize the shape of the conductors in order to allow high deformations without permanent damage [17]. Based on these results, the interconnections between two islands will form a two-dimensional horseshoe-shaped metal track [6]. In this design, stresses are distributed in a wider region instead of being concentrated in a small zone. The horseshoe pattern is created by joining a series of circular arcs. Fig. 3 (bottom) resumes the main characteristics of a horseshoe shape meanders. They are defined by an angle $H$ between the 
Table 1

Characterization results of different patterns for stretchable interconnections [19].

\begin{tabular}{llll}
\hline Shape & $\begin{array}{l}\text { Resistance increase at } \\
\text { mid-elongation }(\%)\end{array}$ & $\begin{array}{l}\text { Mean resistivity } \\
(\Omega / \mathrm{cm})\end{array}$ & $\begin{array}{l}\text { Mean } E_{\max } \\
\text { elongation }(\%)\end{array}$ \\
\hline H0 & 1.76 & 0.94 & 57.53 \\
H30 & 1.19 & 1.86 & 74.82 \\
H45 & 1.28 & 2.23 & 77.68 \\
\hline
\end{tabular}

extremities of the shape and the horizontal line, a width $W$ and a diameter $D$. Shapes with a positive or negative angle of " $x$ " degrees are defined as $H x$ and $H-x$ respectively [18]. H45 has a high maximum elongation, but a higher electrical resistance. HO has a lower maximum elongation, but a lower resistance. $\mathrm{H}-20$ is a stackable structure to reduce the pitch drastically $[17,18]$. As shown in Table 1 ; $\mathrm{HO}, \mathrm{H} 30$ and $\mathrm{H} 45$ have a very low increase of resistivity $(<2 \%)$ during mid elongation. Mean $E_{\max }$ increases from $58 \%$ for $\mathrm{HO}$ up to $78 \%$ for H45 [19]. Depending on the target application, a compromise between a high maximal elongation (hence, reliability) and a low track resistance should be found. In order to increase the reliability of stretchable electronic interconnections, the meanders can be shunted by the deposition of a stretchable conductive polymer layer on top of the metal tracks, which has lower conductivity but higher elongation and reliability. If the metallic meander is broken, the conductive polymer acts like a bridge or a shunt and insures global connectivity $[19,20]$.

The polymer can be silver-filled or an organic conductive polymer (polypyrrole, pedot, polyaniline, etc.), but should be stretchable. Amongst the products available on the market XCS80091-1 from Emerson \& Cumming and DA 6524 from Dow Corning have been tested $[19,20]$. A layer of XCS80091-1 with $300 \mu \mathrm{m}$ width and height was tested by Axisa et al. [19] in association with a $425 \mu \mathrm{m} \mathrm{H} 45$ meander. The resistivity of the compound increases from $0.7 \Omega / \mathrm{cm}$ up to $1.4 \Omega / \mathrm{cm}$, from no deformation up to $100 \%$ extension. It was shown that the compound intervention starts at about $60 \%$ elongation with a slight increment of the joint resistance [19].

The DA 6524 has excellent adhesion to most common substrates, is moisture resistant, support extensions up to $80 \%$ and is characterized by excellent electrical conductivity (volumetric resistivity $0.3 \mathrm{~m} \Omega / \mathrm{cm}$ ). Although the performances are extremely promising, a drawback is certainly its cost: about 400 USD per $36 \mathrm{~g}$ of product, which limits the use of this material to very special cases.

\subsection{Technology 1: "biocompatible technology"}

The process to embed golden meander interconnections in a stretchable material was described in Ref. [18] and is shown in Fig. 4. In step 1, a photoresist layer is spin coated on a copper foil. In step 2 , the photoresist is patterned with the desired conductor shape. Because gold cannot be electroplated directly on the copper and cannot be soldered, the third step involves the electroplating of three layers, instead of one. First a nickel or platinum seed layer, second, a $4 \mu \mathrm{m}$ thick gold layer and third, a Ni-Au finish to allow soldering components on pad areas. Steps 4-7 are then straightforward: remove the photoresist, mould in a stretchable material and cure for $4 \mathrm{~h}$ at $60^{\circ} \mathrm{C}$, remove the copper foil, mould and cure the inverted sample. Silastic MDX4-4210 from Dow Corning has been chosen as the stretchable substrate material for moulding. It is a medical grade silicone elastomer with a low Young's modulus of $0.726 \mathrm{MPa}$ [21], high elongation up to $470 \%$, a viscosity of $60,000 \mathrm{cP}$ and hydrophobic surface. Thicker layers of the silicone can be applied to the areas where components are soldered to give a better protection to excessive strain.

The above-described process was developed in the frame of the Bioflex project (Flemisch Government IWT SBO programme (contract 04101)). Although the technology certainly has some beneficial properties for biomedical applications, so far no complete devices have been implanted for a long time period. In vitro testing has been performed in order to prove how surface treatment of both top and bottom layer of the processed PDMS results in a waterproof sealing [22]. In order to achieve a highly biocompatible system, leaking of metals from the encapsulated device should be avoided [22]. Rather than using adhesive bonding materials, nonthermal plasma treatment of the two layers was used to enhance their adhesion [22]. Plasma treatment of the external surfaces was also shown to be beneficial in order to increase the hydrophilic behavior of the overall structure, therefore promoting the biocompatibility [22,23]. An exhaustive study about the aging effect of the hydrophilic effect is also discussed in Ref. [22].

Although the technology has not been used yet in human body implants, there are examples in literature where flexible silicone based systems have been tested in vivo in cochlear implants [24], neural interfaces [25] and monitoring systems [26,27]. The implant of a PDMS encapsulated bladder monitor in a rabbit, lasting for a few hours, has been described by Coosemans et al. [27].

\subsection{Technology 2: "Fast prototyping technology"}

Fig. 5 contains the process steps of the "Fast prototyping technology". This technology has been described in Ref. [28] and consists of laser cutting meander interconnects directly on a flex-print circuit. First, a flexible board is fixated to a support, using an adhesive (i.e. wax), either locally or on its entire surface. This prevents its movement during processing and ensures the flatness of the flex (Fig. 5(2)). Then the meander shaped interconnections and the functional islands are cut using a YAG laser (Fig. 5(3)) set with the following parameters: Nd at $355 \mathrm{~nm}$ wavelength, $375 \mathrm{~mW}$ of power, a frequency of $10 \mathrm{kHz}$, an ablation speed of $5 \mathrm{~mm} / \mathrm{s}$, a Gaussian beam spot of $\sim 20 \mu \mathrm{m}$ diameter, and a perpendicular angle. In the third step, the excess of material not forming the meanders
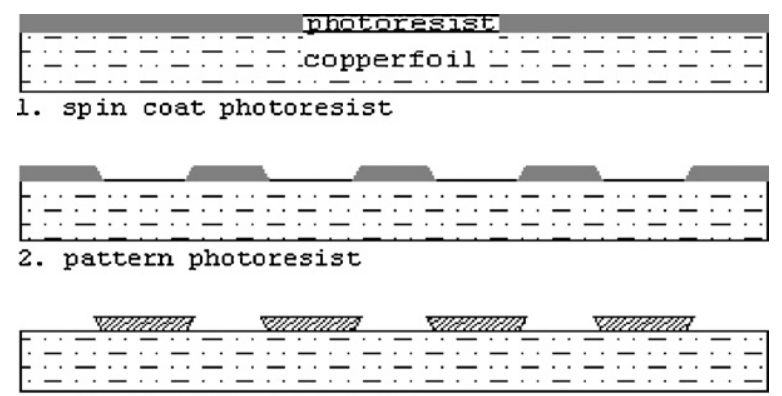

3. electroplate gold metal

4. dissolve photresist

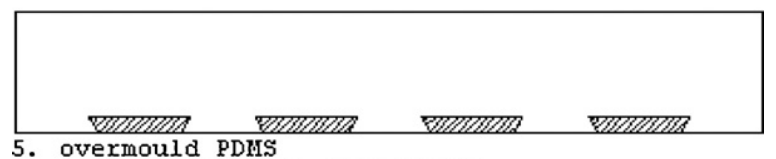

rermould PDos

6. etch sacrificial copperfoil

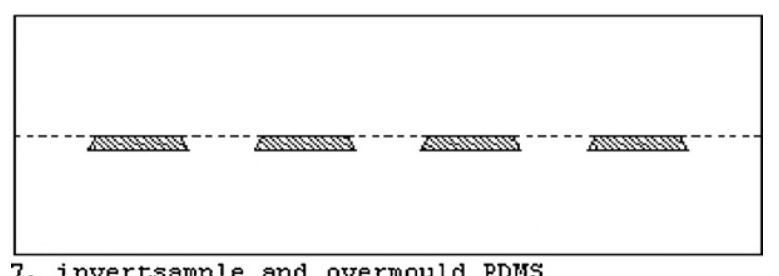

7. invertsample and overmould PDHs

Fig. 4. Process sequence for metallic stretchable interconnections embedded in PDMS [18]. 


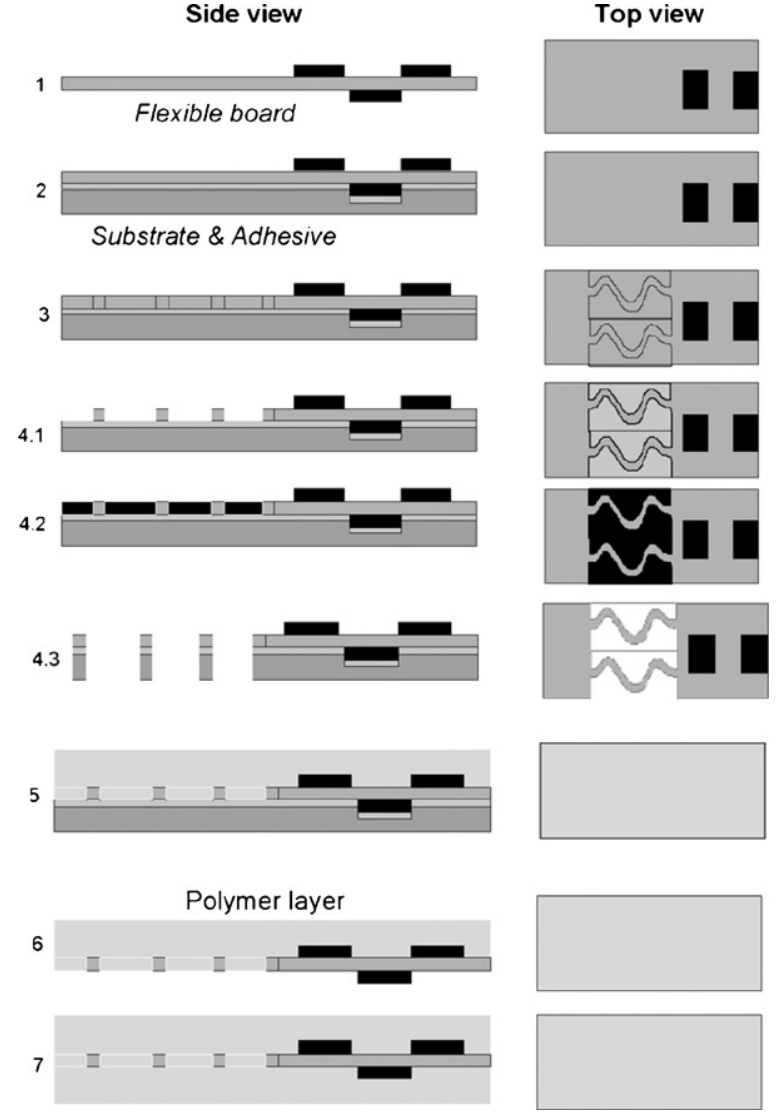

Fig. 5. Process flow to obtain a stretchable electronics board using Nd-YAG laser cutting technology and moulded interconnect device (MID) for encapsulation [28].

is cut off (Fig. 5(4)). Then the top layer of the flexible circuit is embedded in polymer using a moulding, casting or laminating step (Fig. 5(5)). At this stage, the support can be removed by dissolving the adhesive or melting the wax (Fig. 5(6)), the circuit is embedded completely using moulding or casting. Moulded interconnect device (MID) is chosen as it is a reliable way to embed components in a polymer $[6,29]$. The system is encapsulated inside a mould; the polymer is injected and cured at a temperature of $150^{\circ} \mathrm{C}$ for a period of $30 \mathrm{~min}$. The moulding plates are made out of polyetherimide ULTEM $^{\circledR} 1000$, based on its broad chemical resistance, its high heat resistance and its high mechanical strength and rigidity. The result is a system composed of flexible islands interconnected by conductive and stretchable meanders all embedded in a polymer (Fig. 5(7)). This technology allows the production of a stretchable system out of any flexible board. Complex multilayer stretchable electronic boards can also be produced using this technology.

\section{Experimental results}

To explore the limits and potentials of the technology, two simplified demonstrators were designed and produced before approaching the realization of the wireless battery charger. This section describes the realization process of these prototypes, the work towards the realization of the final device and concludes with a discussion on the measurements and testing.

\subsection{Realization of the first demonstrator}

The first demonstrator consists of a flexible circuit cased in silicone as a carrier. This is a single functional island containing a receiving antenna for inductive power transfer, a half bridge rectifier, a voltage regulation module and a LED that is made blinking by applying OOK modulation to the power carrier. The built-in device was realized using a variant of the biocompatible technology [30], using copper instead of gold for the routing and a thickness of $70 \mu \mathrm{m}$ in order to keep the receiving coil's quality factor high. The purpose of this demonstrator is showing the feasibility of the process within a simple application and to test the moisture resistance of the package (Fig. 6).

\subsection{Realization of the second demonstrator}

The second simplified demonstrator is an evolved version of the first one. Besides proving the moisture resistance, it is intended to prove the feasibility of the stretchable interconnects between two functional flexible islands. In this case the first island contains the receiving coil and the conversion electronics whereas the second island contains a MCU which drives a LED array with a user defined pattern. This clustering was chosen as it needs only two interconnections (GND and VDD).

One of the main constraints for the realization of this demonstrator is its size. All fabrication machines of the laboratory (photolithography, lamination) are sized for square 4 in. $(\sim 10 \mathrm{~cm})$ substrates, and the demonstrator is over $10 \mathrm{~cm}$ in size. The dimensions of the demonstrator can be seen in Fig. 7 together with a phase of the production process. Therefore the demonstrators have been processed in three steps:
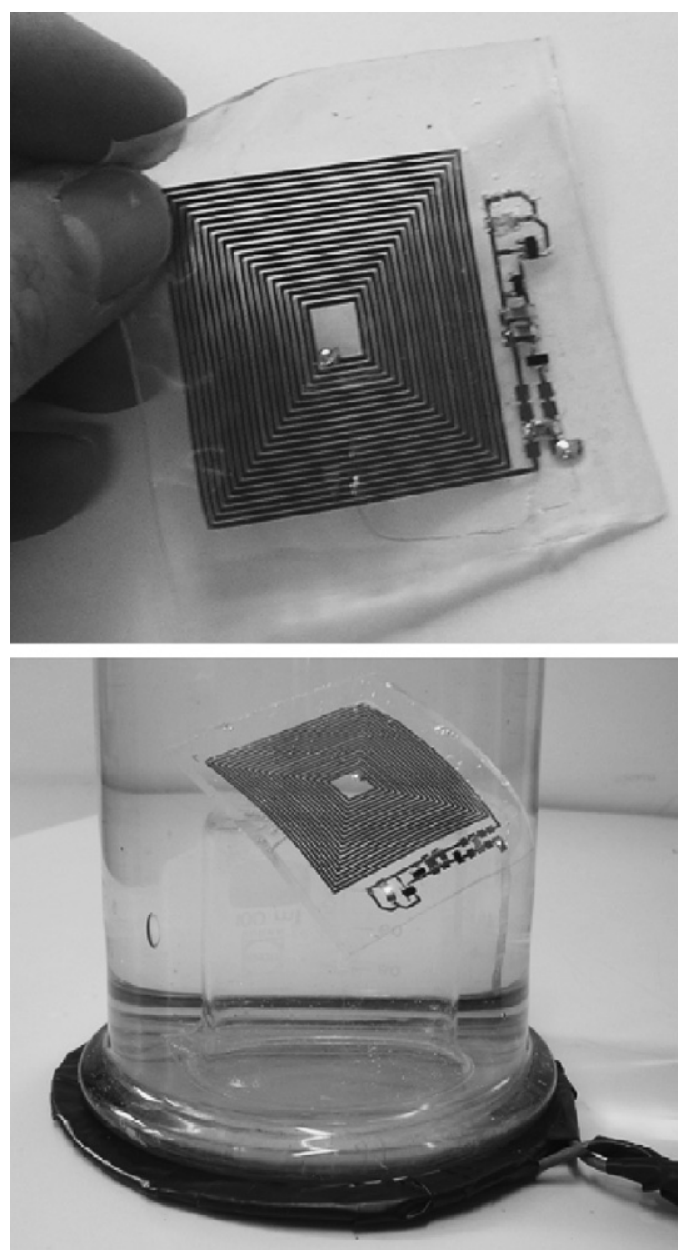

Fig. 6. Simplified wireless power transfer demonstrator: detail (top) and moisture resistance test (bottom). The built-in device consists of a LC resonating tank, a rectifier/regulator stage and a LED which is made blinking by applying OOK modulation to the power carrier. 

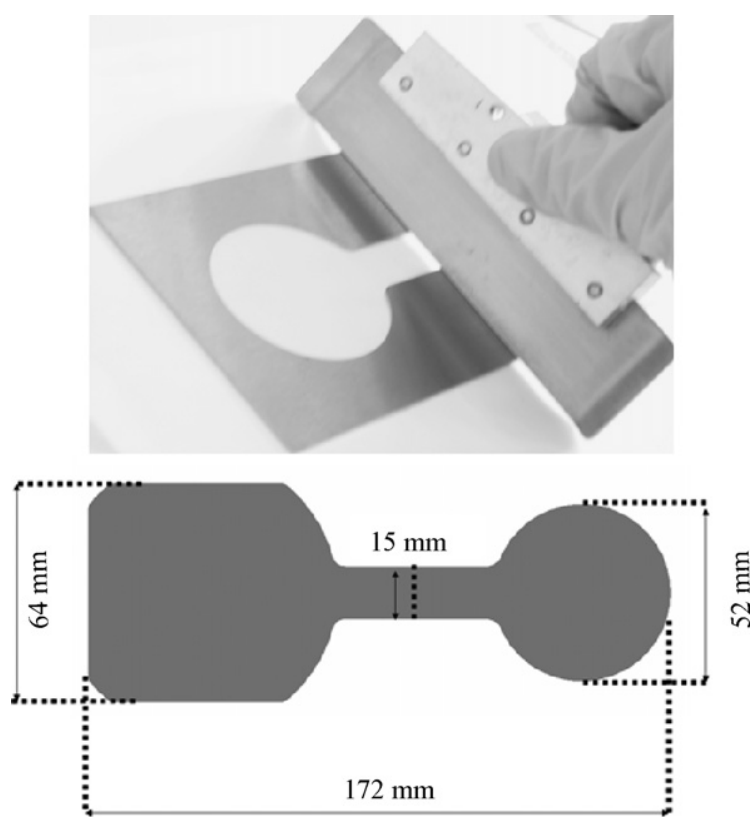

Fig. 7. A realization phase of the simplified prototypes (top) and the dimensions of the second demonstrator (bottom).

First, the two flexible islands are fabricated, following the standard procedure for flexible fabrication. To start, $20 \mu \mathrm{m}$ of polyimide (Upilex 25S) was laminated on a $70 \mu \mathrm{m}$ thick copper foil (TWTW from CircuitFoil, Luxembourg). The thickness of the copper foil was chosen to have a better quality of the secondary coil. Following, photolithography is used before etching with a $\mathrm{CuCl}_{2}$ solution. After this, the solder mask is screen-printed and the components are soldered using a lead free SAC solder paste in a reflow oven. The shunt wire closing the coil is soldered manually, as it is a one-layer flexible electronic board.

Secondly, the interconnections are fabricated in two versions according to the technologies described in Sections 3.2 and 3.3. Both versions will create interconnections which consist of two parallels tracks embedded in a $1 \mathrm{~cm}$ width, $250 \mu \mathrm{m}$ thick and $7 \mathrm{~cm}$ long PDMS band. Each track consists of three parallel sub-tracks connected to the same solder pad at the end of the band. Each sub-track is a $\mathrm{H} 45$ meander with a width of $25 \mu \mathrm{m}$ and an external radius of $82 \mu \mathrm{m}$.

The first version has been processed according to technology 1 "biocompatible technology" with a track thickness of $8 \mu \mathrm{m}$ and is then covered by a screen-printed line of conductive silicone (DA 65-24 from Dow Corning). Each line of conductive silicone has a width of $1.7 \mathrm{~mm}$ and a thickness of $30 \mu \mathrm{m}$ insuring the reliability of the interconnection.

The second version has been processed according to technology 2 "Fast prototyping technology" where the tracks are laser cut from a $18 \mu \mathrm{m}$ thick TW-YE copper foil from CircuitFoil.

In the third step of the fabrication process, the stretchable interconnections are connected to the flexible islands with conductive silicone DA 65-24 from Dow Corning and embedded with $1 \mathrm{~mm}$ metal casting plates. Both top and bottom PDMS layers have a thickness of $1 \mathrm{~mm}$. An adhesion promoter 12000 from Dow Corning is used and a thicker top layer is cast over the areas containing soldered components to protect them against excessive strains.

\subsection{Towards the realization of the final device}

Once the technology was assessed, the realization of a flexiblestretchable advanced system could begin. The layout of the wireless battery charger, described in Section 2, was transferred from a rigid
PCB to a flex realization. The main constraint for the prototype realization is again its dimensions which will first force a multistep process with the separate realization of flexible islands and interconnections, and their assembly and casting afterwards. The critical point will be the link between the second and third island since it requires four stretchable lines (VDD, GND, DATA_IN and DATA_OUT). The reliability of the elastic joints will be of utmost importance to guarantee the correct operation of the system. In this view, the biocompatible process to realize the interconnections is preferred.

\subsection{Measurements and testing}

The first demonstrator was shown to be fully operational for 30 days submerged in tap water. Fig. 6 depicts on the top a detail of the flexible demonstrator and on the bottom a phase of the testing in liquid environment. The magnetic field source is placed under the jar and the embedded LED is blinking.

Two versions of the second demonstrator have been realized (one sample in each of the two technologies). They were both functional right after production. The laser cut sample has failed in the meantime due to mechanical stress. A preliminary conclusion is that at least a better design of the flex-to-stretch transitions is necessary for achieving better resistance to mechanical stress. Ideas for improvement are under investigation and will be published in a separate paper. Fig. 8 depicts the two versions of the second demonstrator, with at the top the prototype realized with the "biocompatible process". The details of the interconnection, consisting of multiple metal tracks with conductive silicone, are illustrated in the magnification (top and bottom views). At the bottom the same demonstrator realized with the "fast prototyping process" is shown. The magnifications again depict a detail of the meander and a joint between the interconnection and functional island.

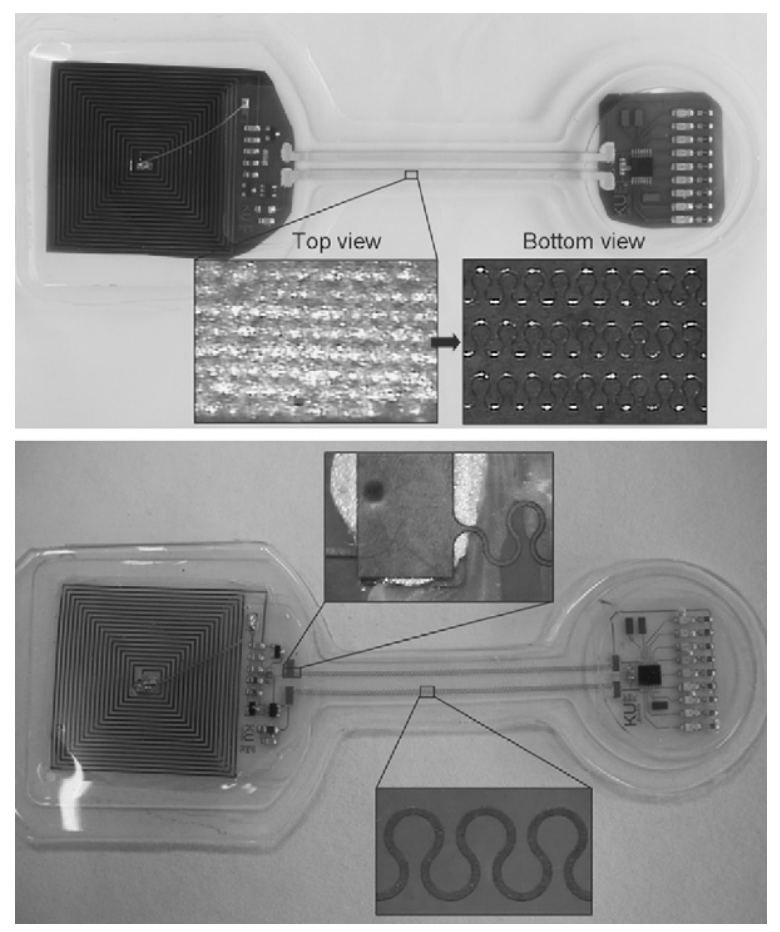

Fig. 8. Demonstrators of the flexible-stretchable platform. At the top: the realization with the biocompatible process; at the bottom: the realization with the fast prototyping process. In both the realizations, the first island (left) contains an LC tank, a rectifier and a regulation stage, the second island (right) contains a $\mu \mathrm{C}$ and a LED array. In this case no data transfer is foreseen trough the link but the LEDs blinking pattern is driven by the controller. 
The three steps realization has to be considered as a preliminary solution due to the lack of equipment at CMST laboratories. This solution was adopted to verify whether the system's functionalities were preserved under mild stress conditions. Further realization will be based on a single step production. The mechanical failure of such a built-in device has been studied in [31] and comes to the conclusion that a decrease of stress concentration can be achieved by gradual flex to stress transitions, mainly by avoiding sharp connection angles in the meander-pad junction. This principle has been applied to the design of the developed systems (detail of Fig. 8 (bottom)).

The wireless battery charger was tested in its PCB and flexprint realization before processing the layout in the flexible-stretchable technology. Fig. 9 depicts a testing phase of the charger. The flexprint has been cut in three parts corresponding to the different functional islands to be connected by elastic joints. For testing purposes standard copper wire was used to link the islands at this stage.

The inductive link supports a power transfer up to $200 \mathrm{~mW}$, it is robust to misalignment and coil distance variation up to $10 \mathrm{~cm}$. Bidirectional data transfer, with a rate fixed at $4.8 \mathrm{kbps}$, is supported during recharge cycles.

When the inductive link is absent, the battery provides power to the MCU which supplies the load. The system works as a standalone station, the array of LEDs display a Ready status, i.e. a blinking pattern of the 8th LED. When the inductive link is present, power to the MCU, load and battery charger is received through the inductive link. The charger starts charging the battery and the LED array displays a thermometric scale of the battery status.

Sending the "Retrieve" command results in the MCU returning two bytes through the uplink channel. The two bytes are respectively the voltage over and the current to the battery. Fig. 10 depicts the transmitted and received signals respectively on the downand uplink. In the top figure, the upper graph is the signal at the primary side before modulation onto the carrier; the lower graph shows the same signal after demodulation at the secondary side. Noise can be seen before and after the signal because the comparator involved in the demodulation process could not compare the signal to a previous state and hence giving a toggling output. This problem will be solved by adding a hysteresis to the comparator input.

The uplink (bottom figure) was tested using a block wave (upper graph). Here, the received signal is shown before demodulation and it represents the voltage variation across the coil $\mathrm{L}_{1}$ (lower graph).

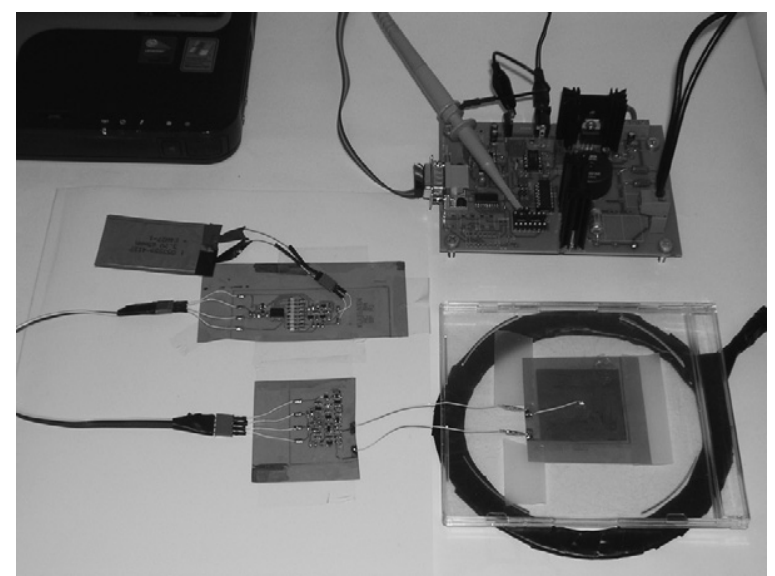

Fig. 9. Testing of the flexible version of the wireless battery charger. The final system consists of three islands and its implementation is in progress using in the MID technology. The first island only contains the power receiving coil, the second one the power conversion electronics and the modulation/demodulation unit, the third contains the $\mu \mathrm{C}$, the battery charger and the LED array.
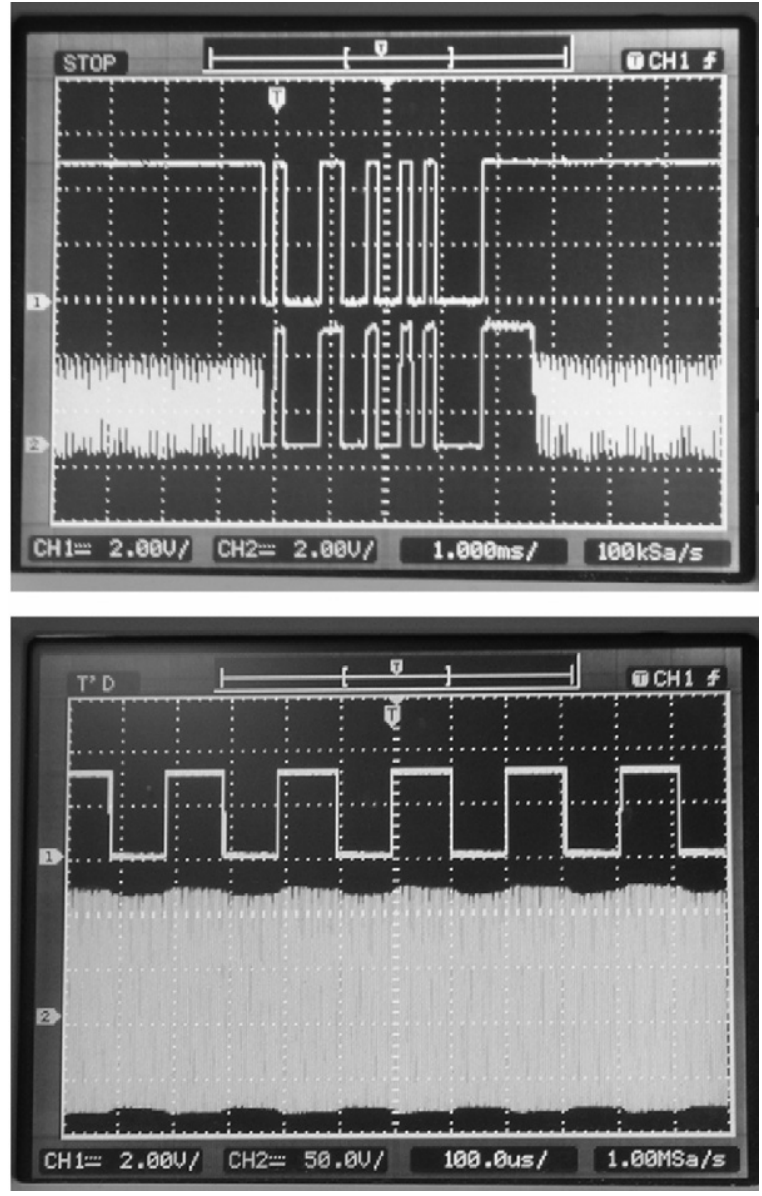

Fig. 10. Wireless battery charger: measurements of transmitted (upper graph) and received (lower graph) data for downlink (top figure) and uplink (bottom figure). The received signal is shown before demodulation for the uplink, after demodulation for the downlink.

\section{Conclusion}

A stretchable-flexible technology platform for the realization of electronics circuits has been proven to be sufficiently reliable to allow the implementation of advanced systems.

Two different technologies have been tested and compared on simple demonstrators in order to evaluate the potential for integration of more advanced systems. Moisture resistance and elastic interconnections of different functional islands were proven. The design of a wireless battery charger is presented and its layout oriented towards the implementation on the stretchable-flexible technology. The system was tested in its flexible realization and the prototype is now in its final production step using the target technology. Robustness of the inductive link, charging of the battery and bidirectional data communication on a flex-and-stretch print realization for integration in clothes, have been demonstrated. The link can provide power up to a maximal distance of $\sim 10 \mathrm{~cm}$ and up to a maximum of $200 \mathrm{~mW}$. A bidirectional data transfer at a fixed rate of $4.8 \mathrm{kbps}$ is guaranteed during the battery recharge.

The system functionality was tested with an integrated microcontroller. Future implementations of this system will involve the integration of sensors, to monitor biomedical parameters such as heart beat, temperature, humidity and respiration rate.

\section{Acknowledgements}

The work is supported by the Belgian Science Policy (Belspo) through the Sweet project (contract number P2/00/08). This work 
is also supported by the Institute for the Promotion of Innovation by Science and Technology in Flanders (IWT) through the SBO-Bioflex project (contract number 04101). The authors wish to thank the project partners and the respective funding organizations.

\section{References}

[1] M. Catrysse, B. Hermans, R. Puers, An inductive power system with integrated bi-directional data-transmission, Sens. Actuators A: Phys. 115 (2004) 221-229.

[2] J. Coosemans, B. Hermans, R. Puers, Integrating wireless ECG monitoring in textiles, Sens. Actuators A: Phys. 130-131 (2006) 48-53.

[3] Nathan M. Neihart, Reid R. Harrison, Micropower circuits for bidirectional wireless telemetry in neural recording applications, IEEE Trans. Biomed. Eng. 52 (2005) 1950-1959.

[4] B. Ziaie, S.C. Rose, M.D. Nardin, K. Najafi, A self-oscillating detuning-insensitive class-E transmitter for implantable microsystems, IEEE Trans. Biomed. Eng. 48 (2001) 397-400.

[5] W. Mokwa, Ophthalmic implants, in: Proceedings of the IEEE Sensors, 2003, pp. 980-986.

[6] F. Axisa, D. Brosteaux, E. De Leersnyder, F. Bossuyt, J. Vanfleteren, B. Hermans, R. Puers, Biomedical stretchable systems using MID based stretchable electronics technology, in: Proceedings of the IEEE-EMBS, 2007, pp. 5687-5690.

[7] D.S. Gray, J. Tien, C.S. Chen, High-conductivity elastomeric electronics, Adv. Mater. 16 (2004) 393-397.

[8] S.P. Lacour, J. Jones, S. Wagner, T. Li, Z. Suo, Stretchable interconnects for elastic electronic surfaces, Proc. IEEE 93 (2005) 1459-1467.

[9] S.P. Lacour, S. Wagner, R.J. Narayan, T. Li, Z. Suo, Stiff subcircuit islands of diamondlike carbon for stretchable electronics, J. Appl. Phys. 100 (2006) 014913.

[10] R. Carta, P. Jourand, B. Hermans, J. Thoné, D. Brosteaux, F. Axisa, J. Vanfleteren, R. Puers, Design and implementation of complex systems on flexible-stretchable technology towards embedding in textile, in: Proc. Eurosensors XXII, 2008, pp. 1384-1387.

[11] M.T. Do, J.-L. Auge, O. Lesaint, Dielectric losses and breakdown in silicone gel, in: Proceedings of the IEEE Conference on Electrical Insulation and Dielectric Phenomena, 2006, pp. 541-544.

[12] K. Otsuka, Y. Takeo, T. Yamada, S. Kuroda, H. Tachi, The mechanisms that provide corrosion protection for silicone gel encapsulated chips, IEEE Trans. Compon. Hybrids Manuf. Technol. 10 (1987) 666-671.

[13] M. Catrysse, R. Puers, C. Hertleer, L. Van Langenhove, H. van Egmond, D. Matthy, Towards the integration of textile sensors in a wireless monitoring suit, Sens. Actuators A: Phys. 114 (2004) 302-311.

[14] C. Omar, F. Kamlin, J.A. Dawson, C.P.F. O’Donnell, C.J. Morley, S.M. Donath, J. Sekhon, P.G. Davis, Accuracy of pulse oximetry measurement of heart rate of newborn infants in the delivery room, J. Pediatr. 152 (2008) 756-760.

[15] J. Van Ham, P. Reynders-Frederix, R. Puers, An autonomous implantable distraction nail controlled by an inductive power and data link, Proceedings of the Transducers (2007) 427-430.

[16] Z. Yu, O. Graudejus, C. Tsay, S.P. Lacour, S. Wagner, B. Morrison, Stretchable microelectrode array: a potential tool for monitoring neuroelectrical activity during brain tissue deformation, J. Neurotrauma 24 (2007), P. 1278 P200.

[17] M. Gonzalez, F. Axisa, M. Vanden Bulcke, D. Brosteaux, B. Vandevelde, J. Vanfleteren, Design of metal interconnects for stretchable electronic circuits using finite element analysis, in: Proceedings of the 8th International Conference on Thermal, Mechanical and Multiphysics Simulation and Experiments in MicroElectronics and Micro-Systems, EuroSimE, 2007.

[18] D. Brosteaux, F. Axisa, M. Gonzalez, J. Vanfleteren, Design and fabrication of elastic interconnections for stretchable electronic circuits, IEEE Electron Dev. Lett. 28 (2007) 552-554.

[19] F. Axisa, D. Brosteaux, E. De Leersnyder, F. Bossuyt, M. Gonzalez, N. De Smet, E. Schacht, M. Rymarczyk-Machal, J. Vanfleteren, Low cost, biocompatible elastic and conformable electronic technologies using MID in stretchable polymer, in: Proceedings of the IEEE-EMBS, 2007, pp. 6592-6595.

[20] F. Axisa, F. Bossuyt, J. Missine, R. Verplancke, T. Vervust, J. Vanfleteren, Engineering technologies for the development of advanced stretchable polymeric systems, in: Proceedings of Portable-Polytronic, 2008, pp. 1-8.

[21] S. Reichelt, J. Fiala, A. Werber, K. Förster, C. Heilmann, R. Klemm, H. Zappe, Development of an implantable pulse oximeter, IEEE Trans. Biomed. Eng. 55 (2008) 581-588.

[22] R. Morent, N. DeGeyter, F. Axisa, N. DeSmet, L. Gengembre, E. DeLeersnyder, C. Leys, J. Vanfleteren, M. Rymarczyk-Machal, E. Schacht, E. Payen, Adhesion enhancement by a dielectric barrier discharge of PDMS used for flexible and stretchable electronics, J. Phys. D: Appl. Phys. 40 (2007) 7392-7401.

[23] R.L. Williamsa, D.J. Wilsona, N.P. Rhodesa, Stability of plasma-treated silicone rubber and its influence on the interfacial aspects of blood compatibility, Biomaterials 25 (2004) 4659-4673.

[24] F.-G. Zeng, S. Rebscher, W. Harrison, X. Sun, H. Feng, Cochlear implants: system design, integration, and evaluation, IEEE Rev. Biomed. Eng. 1 (2008) 115-142.

[25] K.P. Hoffmann, K.P. Koch, T. Doerge, S. Micera, New technologies in manufacturing of different implantable microelectrodes as an interface to the peripheral nervous system, in: Proceedings of the BioRob, 2006, pp. 414-419.

[26] B.D. Ratner, et al., Biomaterials Science: An Introduction to Materials in Medicine, 2nd edition, Elsevier Academic Press, 2004, ISBN 0125824637.
[27] J. Coosemans, Wireless and battery-less medical monitoring devices, Ph.D. Thesis, K.U. Leuven, Belgium, 2008.

[28] F. Axisa, F. Bossuyt, T. Vervust, J. Vanfleteren, Laser based fast prototyping methodology of producing stretchable and conformable electronic systems, in: Proceedings of the ESTC, 2008, pp. 1387-1390.

[29] F. Axisa, D. Brosteaux, E. De Leersnyder, F. Bossuyt, M. Gonzalez, M. Vanden Bulcke, J. Vanfleteren, Elastic and conformable electronic circuits and assemblies using MID in polymer, in: Proceedings of the Polytronics, 2007, pp. 280-286.

[30] F. Axisa, J. Vanfleteren, T. Vervust, Method for manufacturing a stretchable electronic device, US Patent Application, Serial No. 12/145,369, filed on June 24 2008.

[31] M. Gonzalez, F. Axisa, F. Bossuyt, Y.-Y. Hsu, B. Vandevelde, J. Vanfleteren, Design and performance of metal conductors for stretchable electronic circuits, in: Proceedings of the ESTC, 2008, pp. 371-376.

\section{Biographies}

Riccardo Carta was born in Cagliari, Italy, in 1978. In 2004 he received his M.Sc degree in electrical engineering, with major in Microelectronics, from the University of Cagliari, Italy. Currently, he is a research assistant at the Microelectronics and Sensors (MICAS) group of the Katholieke Universiteit Leuven, Belgium, where he is working towards a Ph.D. degree. His main research interest is in the area of wireless power and data transmission for biomedical applications.

Philippe Jourand was born in 1982 in Leuven, Belgium. He obtained his masters degree in electrical engineering in biomedical techniques in 2007 at the Katholieke Universiteit Leuven, Belgium. From 2007 up to now, he is currently working towards obtaining a Ph.D. in electrical engineering at the MICAS group of the Katholieke Universiteit Leuven, Belgium. His main research is focused on creating system interfaces for portable and implantable sensor systems for biomedical applications.

Bart Hermans was born in Vilvoorde, Belgium, in 1977. He received his B.S. degree in electro-mechanics from K.I.H. Denayer in 1999. In 2001, he received his M.S. degree in electrical engineering at the Katholieke Universiteit Leuven. Currently, he is a research assistant at the ESAT-MICAS Laboratories of the Katholieke Universiteit Leuven, where he is working towards a Ph.D. degree. His main research interests are inductive powering, low power data transmission for biomedical applications and intelligent implantable monitoring systems. In 2007 he founded a spin-off company ZENSO NV. ZENSO is a design house for electronic engineering.

Jef Thoné received his bachelor degree in automotive technology in 1999 from the Hogeschool voor Wetenschap en Kunst in Schaarbeek, Belgium. In 2002 he received his Master of Engineering degree in electronics at the Karel de Grote Hogeschoo in Hoboken, Belgium. From 2002 to 2006 he was employed as ASICasic designer and project manager at Melexis Belgium, where he cooperated in the analog design of several automotive sensor interface ASICs. In October 2006 he joined the Sensor Systems Group of MICAS. His Ph.D. research focuses on high-data rate biocompatible telemetry systems. His research interests include sensor interfacing, analog IC and discrete design, FPGA and microcontroller interfacing, electronic system design and prototyping.

Dominique Brosteaux graduated in 2003 as physical engineer at Ghent University Since April 2005 he is working on a doctorate as a member of CMST at the Electronics and Information Systems Department of Ghent University.

Thomas Vervust was born in Brugge (Belgium) on April 30, 1984. He obtained his master degree in electronics engineering from the University of Gent in 2007. From 2007 on, he works as a Ph.D. student at the Laboratory of Electronics of the University of Gent, department Center for MicroSystem Technologies, where he is involved in research on Stretchable and washable electronics for embedding in textile.

Frederick Bossuyt was born in Kortrijk (Belgium) on September 15, 1983. He obtained his degree in electronics engineering from the University of Gent in 2006. From 2006 on, he works as a Ph.D. student at the Laboratory of Electronics of the University of Gent, Department Center for MicroSystem Technologies, where he is involved in research on Stretchable Electronics Technologies.

Fabrice Axisa was born in Toulouse (France) on April 13, 1974. He obtained his engineering degree in microelectronics, microsystems and engineering at Ecole Centrale de Lyon in 1996 and a Ph.D. in biomedical systems, microsensors and microsystems at INSA de Lyon, France, in 2004. From 1997 to 2001, he was working as a micro electronic engineer for ARM and Texas Instruments, Sophia Antipolis, France. He is currently working for IMEC (CMST Department) in Belgium, and he is focusing on stretchable electronics for biomedical systems, for smart textile and more generally in stretchable engineering technologies.

Jan Vanfleteren obtained his Ph.D. in Electronic Engineering from Ghent University, Ghent, Belgium in 1987. He is currently a senior engineer and project manager at IMEC in the Centre for Microsystems Technology and is involved in the development of novel interconnection, assembly and substrate technologies, especially in the field of flexible and stretchable electronics. In 2004 he was appointed as part time professor at Ghent University. Prof. Dr. Vanfleteren is a member of IMAPS and 
IEEE and (co)-author of over 100 papers in international journals and conferences and he holds 6 patents/patent applications.

Robert Puers received his B.S. degree in electrical engineering in Ghent in 1974, and his M.S. degree at the Katholieke Universiteit Leuven in 1977, where he obtained his Ph.D. in 1986. In 1986 he became Director (NFWO) of the clean room facilities for silicon and hybrid circuit technology at the ESAT-MICAS laboratories of the same University. He was a pioneer in the European research efforts in silicon micro machined sensors, MEMS and packaging techniques, for biomedical implantable systems as well as for industrial devices. In addition, his general interest in low power telemetry systems, with emphasis on low power intelligent interface circuits and on inductive power and communication links has promoted the research of the ESAT-MICAS lab- oratory to international recognition. At present, he is a full professor at the K.U. Leuven, teaching courses in 'Microsystems and Sensors', in 'Biomedical Instrumentation and Stimulation', in 'Biomedical Equipment and Regulations', in 'Production techniques for microelectronics', and basic courses in 'Electronics, System Control and Information Technology'. He is the author or co-author of more than 400 papers on biotelemetry, sensors, MEMS and packaging in reviewed journals or international conferences. He is a Fellow of the Institute of Physics (UK), council member of the International Microelectronics and Packaging Society (IMAPS), member of the Electron Device Society (EDS) and many others. He is Editor-in-Chief of the IOP Journal of Micromechanics and Microengineering, and acted as the General Program Chairman for Transducers'07 in Lyon. 